\title{
Persuasive Decision Support: Improving Reliance on Decision Aids
}

\author{
Alison Parkes \\ Department of Accounting, \\ The University of Melbourne, Melbourne, Australia \\ aparkes@unimelb.edu.au
}

\begin{abstract}
The primary role of a decision aid is to guide and support a decision maker. As reliance on a decision aid is largely discretionary the persuasiveness of the system becomes critically important. In this paper characteristics thought to affect systems persuasiveness are examined. This paper asserts that the target and source of a decision support message, along with the design of the message itself, act to influence the persuasiveness of the decision support provided. Using a purpose built experimental platform with seventy subjects the research finds that the persuasiveness of a decision support message is varied by the perceived difficulty of the task being undertaken, and the perceived usefulness of the decision support provided. The type of decisional guidance provided also affects persuasiveness of the system; in particular, providing suggestive decisional guidance is shown to significantly improve system persuasiveness. The implications of these findings relate to the appropriate design of decision aids, and the contexts within which a decision aid can be expected to persuade decision makers to reply on the support provided.
\end{abstract}

Keywords: Decision support, persuasiveness, decisional guidance, task difficulty, perceived usefulness 


\section{Introduction}

The role of effective decision support is "to guide and direct the decision-maker towards a better solution" (Todd and Benbasat 1999:356), however unlike most other computer systems the use of a decision aid is frequently discretionary. If a decision maker under uses or avoids a decision aid it provides little or no value (Davern and Kauffman 2000). Given that use is optional an important characteristic for decision support is persuasiveness of the system. A persuasive decision aid convinces a decision maker to rely on the decision support provided.

The definition of persuasiveness used is taken from work by Hovland (Hovland 1957; Hovland et al. 1982), exploring persuasive messages. Hovland's theories of persuasion tell us that while persuasion cannot alter personality variables, it can alter attitudes, especially in response to some form of communication. This has important implications for decision support, suggesting that the success or otherwise of a decision aid has causal roots far broader than simply the design of the system itself. Successful decision support potentially encompasses the nature and form of the communications provided by that system, and the intended target of those system outputs.

Hovland (1982) argues that three main factors affect the persuasiveness of a message: the characteristics of the person who receives and processes the message (the target), the credibility of the source of the message (the source) and the nature of the message itself (the message). Although Hovland's work explored human interactions, ideas relating to persuasiveness have been expanded into several information systems contexts.

Jiang et al (2000) found that the target, message and source characteristics of communications all contributed to the persuasiveness of an expert system. Artificial intelligence and recommender agent research also has shown a growing interest in persuasive technologies. A comprehensive review of recommendation agents examined the use and impact of these agents (Xiao and Benbasat 2007). They note that providing explanations for recommendations improves trust, and that improved perceptions of usefulness of the recommender agent can be tied back to design choices such as navigational paths, layout and interface design. Recent IS publications describe persuasive technologies in domains as diverse as healthy eating habits (Mazzotta et al. 2007) and environmental sustainability (Midden et al. 2008). These prior studies provide some expectation that theories of persuasiveness originally framed in a human context will translate meaningfully to information systems contexts.

The approach this paper takes is to examine how the characteristics of the target, source, and message provided by a decision aid affect the persuasiveness of that decision aid; evidenced by the degree to which a decision maker chooses to rely on the decision aid. The paper focuses on perceived task difficulty as characteristic of the target, and perceived usefulness of the decision aid as characteristic of the source. The characteristics of the message itself are explored by adopting concepts from the decisional guidance literature (Silver 1990; 1991). The research question addressed by this paper is "What impacts the persuasiveness of decision aids?"

The structure of the remainder of the paper is as follows: the next section describes the theoretical basis of the work and presents the hypotheses. The methods employed and results obtained are then presented. In the final section of the paper these results are discussed, along with the conclusions reached and their related implications.

\section{Theory and Hypotheses}

\section{Persuasiveness}

"A Decision support system cannot successfully achieve its objectives if it is never used" (Silver 1990:54). Existing theories of technology usage (e.g. Davis et al. 1989; Mathieson 1991) explain physical usage of the decision aid, which is a necessary, but not sufficient, precursor to reliance. Reliance conceptually extends beyond use of the decision aid to include the influence of that decision aid on 
the decision maker. Reliance is more broadly concerned with how decision aid outputs are used and integrated into decision-making (Arnold and Sutton 1998; Hampton 2005). Decision aid use can be organizationally mandated; however reliance, as a matter of course, cannot. In this study reliance is adopted as a proxy for persuasiveness, ceteris paribus a more persuasive decision aid will induce a decision maker to increase their reliance on that system.

\section{Message target characteristics}

Hovland's theories $(1957 ; 1982)$ propose three factors that affect the persuasiveness of a message. The first factor relates to the characteristics of the person receiving and/or processing the message, examples include intelligence and self esteem. In the current study the message target is characterized by the perceived difficulty experienced by the decision maker undertaking the decision task. The complexity of a task is calibrated independently of the decision-maker (Wood 1986); the difficulty of a task is determined by reference to that decision-maker (Van de Ven and Delbecq 1974). A task may contemporaneously be perceived as difficult by one decision-maker and not difficult by another, supporting the claim that perceived task difficulty is characteristic of the message target.

Simon (1955) suggests that due to the amount of effort required to achieve rational thought humans are often incapable of employing optimal decision processes i.e. they have a bounded rationality. As a result humans tend to satisfice, or use simplifying strategies, to resulting in a less than optimal but acceptable decision. As a consequence of this bounded rationality, decision-makers encountering high levels of task difficulty will be more readily persuaded to employ effort accuracy tradeoffs to reduce load to a manageable level (Payne et al. 1993). These effort accuracy tradeoffs occur in proportion to the perceived difficulty of the task being undertaken. Where a decision maker perceives a task as not difficult they would be unlikely to benefit from use of the decision aids, hence the level of system persuasiveness would be low. As perceived task difficulty increases the desirability of use of the decision aid will also increase, due to the increased persuasiveness of the systems in terms of ability to reduce effort whilst maintaining accuracy. It is anticipated that the more difficulty the decision maker experiences when performing the task, the more likely it is that this difficulty will persuade the decision maker to rely on a decision aid, in a manner similar to the effort accuracy tradeoffs discussed earlier (Johnson \& Payne 1985). This relationship between message target characteristics and persuasiveness is hypothesized as:

H1: Decision aid persuasiveness is positively influenced by the perceived difficulty of the decision task being undertaken.

\section{Message source characteristics}

The second persuasiveness factor identified by Hovland $(1982 ; 1957)$ relates to the characteristics of the source of the message; example characteristics include expertise, trustworthiness, attractiveness, credibility. In this study the attractiveness and credibility of the message source is characterized by the perceptions of the usefulness of the decision aid. Perceived usefulness reflects the degree to which the user believes the system will improve their task performance (Davis 1989); and is a well established predictor of intention to use the system. Considering effort accuracy tradeoffs (as discussed previously in relation to message target characteristics), Todd and Benbasat (1994) found that decision aids which make a decision less effortful have a greater chance of altering the decisionmakers decision strategy, with decisionmakers electing to use the decision support and conserve cognitive effort (Todd and Benbasat 1992). Where a decision maker perceives a decision aid as useful they will be more readily persuaded to conserve effort by relying on that decision aid. The relationship between message source characteristics and persuasiveness is hypothesized as:

H2: Decision aid persuasiveness is positively influenced by the perceived usefulness of the decision aid. 


\section{Message Characteristics}

The third and final factor explored by Hovland $(1982 ; 1957)$ relates to the persuasive nature of the message itself. In this study the message is characterized by the form of decisional guidance incorporated into the message design. Silver (1988) suggests that the level and amount of guidance provided by a system can have a major impact on the decision-making process. Silver (1988) proposes incorporation of deliberate guidance mechanisms, which act to motivate users of decision aids to modify their decision process, by relying on the guidance provided. In a similar manner to the role of explanations (as discussed by Xiao and Benbasat 2007) a key issue for decision support is whether a decision aid has mechanisms in place to effectively guide inputs and positively affect the outputs used by the decision-maker (Silver 1991). Thoughtful incorporation of decisional guidance can improve the persuasiveness of the decision aid, producing more effective decision-making. Silver (1991:107) defines decisional guidance as "how a decision support system enlightens or sways its users as they structure and execute their decision making processes." Every decision aid, whether explicitly or implicitly, provides decisional guidance. In this study the decision aid provides explicit decisional guidance.

Prior research has found decisional guidance to be an important explanatory variable in understanding decision maker behavior (Silver 1991; Montazemi et al. 1996; Wilson and Zigurs 1999; Jiang and Klein 2000; Limayem and Desanctis 2000; Parikh et al. 2001; Mahoney et al. 2003). Decisional guidance can provide support for decision-makers in differing ways. Silver (1990) suggests that there is a choice to be made between suggestive guidance (swaying a decision-maker by making recommendations) and informative guidance (enlightening decision-makers by providing them with unbiased pertinent information).

A single decision aid may contain both forms of decisional guidance, and either, or both, may be provided at any decision point. The decision aid used in this research delivered either informative or suggestive decisional guidance to the decision maker. Silver (1990) notes that "Designers who seek to influence decision-makers will usually provide specific suggestions, but they may also do so by providing carefully selected informative guidance. Designers who seek to support but not influence decision-makers may rely heavily on informative guidance, but may also offer some suggestive guidance" (Silver 1990:60).

This research explores characteristics that influence a decision maker to rely on the decision support provided, it is anticipated that providing decisional guidance will impact the persuasiveness of the decision aid message. Considering informative guidance first, providing additional information cues with the message is not expected to affect the persuasiveness of the support offered, given that the primary role of informative guidance has been shown to support, rather than influence, the decision maker. Providing informative decisional guidance is not expected to improve decision aid persuasiveness. By comparison, suggestive guidance which dynamically provides a rational suggestion based on the decision makers prior inputs will increase the ability of the decision aid to persuade the decision maker to rely on the advice offered. Stated as the final hypothesis this becomes:

H3: Decision aid persuasiveness is positively influenced by the provision of suggestive decisional guidance.

To summarize, the persuasiveness of a decision aid is hypothesized to relate to the perceived difficulty of the decision task (the message target), the perceived usefulness of the decision aid (the message source), and the form of decisional guidance supplied (the message itself).

\section{Method}

\section{Experiment design and procedure}

The hypotheses were tested in a laboratory based experimental setting. Participants were final year students who were recruited by the author by means of an announcement at the commencement of lectures. Students were all 
Table 1 - Subject Distribution

\begin{tabular}{|l|l|l|l|l|}
\hline & Informative guidance & Suggestive guidance & No guidance & Total \\
\hline Subjects & 24 & 23 & 23 & 70 \\
\hline
\end{tabular}

accounting or finance majors in the final year of their degree program. The author was not involved in any teaching or assessment for the classes from which students were recruited. Recruitment was timed so that the students had just completed a directly relevant section of the course, covering the formation of going concern opinions. Subjects were on average 23 years old, had 1.5 years work experience, and no insolvency related work experience. These novice participants are representative of the expected users of this type of decision support, which is typically provided as a means of training new entrants into a profession. Providing decision support for a highly experienced professional would be less beneficial due to their existing expertise reducing the value of the support offered.

An additional rationale behind selection of a novice cohort was that it provided an opportunity to limit variability in terms of any preexisting notions of what would constitute suitable decision support for the decision task being undertaken. By removing any prior knowledge of the problem domain all participants were starting from the same point of zero knowledge or task expertise; helping to more accurately isolate the effect of the decision aid.

On entering the laboratory each subject was supplied with the experimental materials and asked to complete the demographics section of the questionnaire. They were shown a demonstration of the DSS, and completed a short tutorial case. Subjects opened a sealed envelope, read through the case study and recorded an unaided decision. Subjects were then directed to make use of the system and record a second (aided) decision. All subjects completed the case in the same way, and then answered a short series of questions related to the case and the decision aid.

Seventy subjects successfully completed the experiment. Subjects attended one of six experimental sessions and were randomly allocated to one of the three treatment groups, as shown in table 1. All experimental sessions used identical scripts and procedures ${ }^{1}$. After finalizing the operationalization and instruments for the study, a participant questionnaire was developed. The questionnaire was paper based as it was felt that requiring participants to answer a screen based questionnaire while concurrently using the decision aid had the potential to confound both the perceived task difficulty and reliance observations. Following development and testing of the software, case studies, and questionnaire, a pilot study was conducted to test the face validity and operation of these materials. The pilot study also served to test the design, sequencing, and timing of the experimental session. As a result of the pilot study minor changes were made to materials (e.g. font size on screens increased, paper materials presented in booklet form etc) prior to undertaking the main study.

\section{Overview of the case study and deci- sion task}

The case study contains appendix materials collected by Arnold et al (2003). The case narrative was developed and written by the author based on the data contained in these appendices. Case development included the creation of a narrative, and identification and appropriate insertion of information cues. The information cues were designed to map directly to the decision model contained in the decision aid. Additional original material was devised by the author to provide information cues not considered in the existing appendix materials.

The case study organization was portrayed as operating in a high tech industry and providing electronic messaging systems. A new product was identified as being currently in the final stages of development but not yet available for sale. The case was designed to

\footnotetext{
${ }^{1}$ Sensitivity analysis was conducted; the lab session attended had no significant effect on any of the variables.
} 


\begin{tabular}{|c|c|c|c|}
\hline & Item & Definition & Item Loading \\
\hline R1 & $\begin{array}{l}\text { I used InsolveDG to assist with my decision mak- } \\
\text { ing. }\end{array}$ & $\begin{array}{l}\text { Use of the system in deci- } \\
\text { sion-making }\end{array}$ & .781 \\
\hline R2 & $\begin{array}{l}\text { My decision was influenced by the recommenda- } \\
\text { tion made by InsolveDG. }\end{array}$ & $\begin{array}{l}\text { How much weight recom- } \\
\text { mendations are given }\end{array}$ & .875 \\
\hline R3 & I followed recommendations made by InsolveDG. & $\begin{array}{l}\text { How much users follow } \\
\text { recommendations }\end{array}$ & .787 \\
\hline R4 & $\begin{array}{l}\text { I altered my decision making process when using } \\
\text { InsolveDG. }\end{array}$ & Integration of outputs & .687 \\
\hline R5 & I used information provided by InsolveDG & Use of the systems outputs & .832 \\
\hline R6 & $\begin{array}{l}\text { I followed recommendations made by InsolveDG } \\
\text { that differed from my personal opinion. }\end{array}$ & $\begin{array}{l}\text { How much users follow } \\
\text { recommendations. }\end{array}$ & .740 \\
\hline \multicolumn{3}{|c|}{ Total variance explained } & $61.8 \%$ \\
\hline
\end{tabular}

ensure it provided a challenge for decision makers, therefore ensuring a longer interaction with the decision aid and providing ample opportunity to observe the effects of the decisional guidance provided. The face validity and complexity of the case study was confirmed by three insolvency experts prior to use in the main experiment.

The context for the study was insolvency decision making where a decision must be made about the future of a company; whether to liquidate the distressed business or to trade-out of its present difficulties. The study used a purpose-built decision aid known as Insolve-DG.

\section{Overview of the decision aid}

Insolve-DG is a decision aid purpose built for a program of behavioral research about the effects of decisional guidance on decision making behavior. It incorporates an underlying decision model and materials gathered in an extensive knowledge acquisition effort that had led to the original INSOLVE system (Collier et al. 1999; Leech et al. 1999; Arnold et al. 2004a; Arnold et al. 2004b), but in all other respects is an independent and distinct artifact. The decision model in Insolve-DG was validated by three expert insolvency practitioners, who indicated that the model accorded with their 'real world' view of the insolvency decision making process.

\section{Operationalizing reliance}

The reliance measure used was a multi item construct, containing six items self assessed by participants. Details of the reliance scale are contained in table 2. The items contained in a prior reliance study (Hampton 2005) formed a starting point for the operationalization, along with the reliance definitions used in this research. Factor analysis showed all 6 items loaded onto a single factor (as shown in Table 2) which, taken in conjunction with a Cronbach alpha value of .88 , indicates that the scale has sufficiently internal validity to support the use of the items as a single construct. The reliance measures were taken immediately after using Insolve-DG for decision making while completing the case study.

\section{Operationalizing perceived task diffi- culty}

Perceived task difficulty was also selfassessed by participants. Existing perceived task difficulty measures were examined with the intention of re-using a previously calibrated instrument. The items adapted for the study were based on work by Van de Ven and Delbecq (1974) and Van de Ven and Ferry (1980) and are shown in Table 3. Factor analysis showed that these three items loaded onto a single factor, individual item loading values are disclosed in Table 3 . In additional testing a Cronbach alpha value of .74 was obtained, indicating that the scale has sufficient internal validity to support the use of the items as a single factor.

\section{Operationalizing perceived usefulness}

Perceived usefulness was measured using the existing six item validated instrument 
(Davis 1989), see table 4 for details. Consistent with previous analyses using this instrument, exploratory data analysis showed all six items loading onto a single factor as shown in Table 4. A Cronbach alpha value exceeding .8 was also calculated, indicating that the scale had good internal validity.

Factor analysis of the items contained in the perceived task difficulty, and perceived usefulness scales revealed that the items loaded onto the relevant factors, establishing the discriminate validity of the construct measures. Principal component analysis found two components with Eigen values exceeding 1 for this set of measures; these two components explained $72 \%$ of the variance observed. The items for perceived task difficulty and perceived usefulness each loaded onto a single component, with no substantial cross loadings to the other component, as reported in Table 5.

\section{Operationalizing decisional guidance}

For experimental purposes several versions of Insolve-DG were created; differentiated only by the form of decisional guidance supplied. The underlying decision model was identical in all versions of Insolve-DG. Suggestive guidance was operationalized by leveraging the hierarchical structure of the decision model. Specifically, where multiple underlying factors contributed to an interim decision, the opportunity existed to ask users directly for an interim decision and provide suggestive guidance as to the interim decision. After extensive modeling exercises, an additive model was found to be the most effective way of generating the suggestive guidance. Figure 1 shows an example of underlying factors and resulting suggestive guidance. Informative guidance in the form of definitional text was embedded into appropriate questions as illustrated in figure 2 .

\section{Table 3 - Perceived task difficulty scale items}

\begin{tabular}{|l|l|l|}
\hline PTD1 & $\begin{array}{l}\text { Difficult problems often arose during this task for which there was no } \\
\text { immediate or apparent solution. }\end{array}$ & .755 \\
\hline PTD2 & I spent a lot of actual thinking time trying to solve this problem. & .784 \\
\hline PTD3 & The Message Wings case was very difficult for me. & .568 \\
\hline Total variance explained & $50.28 \%$ \\
\hline
\end{tabular}

\begin{tabular}{|l|l|l|}
\hline \multicolumn{2}{|l|}{ Table $\mathbf{4}$ - Perceived usefulness scale items } & \\
\hline & Item & Item loading \\
\hline PU1 & Using InsolveDG helped me to accomplish the task more quickly & .904 \\
\hline PU2 & Using InsolveDG improved my task performance & .931 \\
\hline PU3 & Using InsolveDG increased my productivity & .916 \\
\hline PU4 & Using InsolveDG enhanced my effectiveness on the task & .877 \\
\hline PU5 & Using InsolveDG made it easier to do this task & .927 \\
\hline PU6 & I found InsolveDG useful in this task & .840 \\
\hline Total variance explained & $80.99 \%$ \\
\hline
\end{tabular}

Table 5 - Confirmatory Factor analysis

\begin{tabular}{|l|l|l|}
\hline & Perceived Usefulness & Perceived Task Difficulty \\
\hline PTD1 & .082 & $\mathbf{. 7 5 0}$ \\
\hline PTD2 & -.036 &. $\mathbf{7 5 5}$ \\
\hline PTD3 & .056 &. $\mathbf{5 9 7}$ \\
\hline PU1 & .898 & .129 \\
\hline PU2 & .927 & .082 \\
\hline PU3 & .907 & .195 \\
\hline PU4 &. $\mathbf{8 7 4}$ & .085 \\
\hline PU5 & .933 & -.073 \\
\hline PU6 &. $\mathbf{8 4 8}$ & -.116 \\
\hline \% of Variance explained & 54.32 & 17.12 \\
\hline
\end{tabular}




\section{Figure 1 - Suggestive guidance operationalization}

\section{Underlying factors: Question 3. Will the practitioner get paid?}

3.1 Will there be sufficient funds to pay the practitioners fees and ongoing expenses?

3.2 Is a challenge to the practitioner's priority to receive payment of their fees and expenses likely?

\section{InsolveDG}

Will the practitioner get paid?

Based on your previous responses it seems likely that you will be paid, therefore InsolveDG recommends that you should answer Yes here

OYes ONo ONot Known

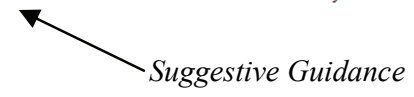

OK

Figure 2 - Informative guidance operationalization

\section{InsolveDG}

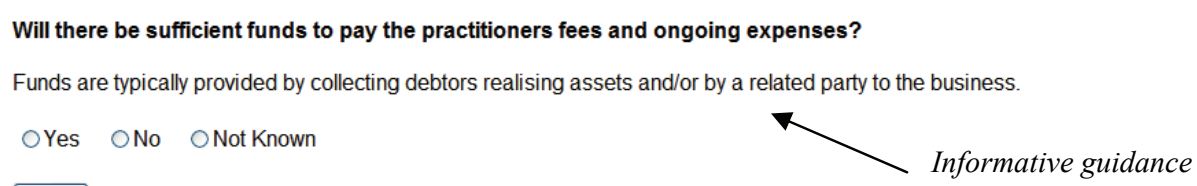

\section{Model estimations}

As the dependent variable and the majority of the independent variables are continuous measures all hypotheses are tested using a linear regression model. The model tests the effects of decisional guidance (message design), perceived usefulness (message source), and perceived task difficulty (message target) on reliance (persuasiveness).

Reliance $=\beta 0+\beta 1 P T D+\beta 2 P U+\beta 3 S G+\varepsilon$

where PTD is a continuous variable for perceived task difficulty

PU Is a continuous variable for perceived usefulness

SG Is a dummy variable for suggestive guidance (1=suggestive guidance, $0=$ otherwise)

For $\mathrm{H} 1$ to be supported $\beta 1$ must be significant to evidence an effect for perceived task difficulty. For $\mathrm{H} 2$ to be supported $\beta 2$ must be significant to evidence an effect for perceived usefulness. For $\mathrm{H} 3$ to be supported $\beta 3$ must be significant to evidence an effect for suggestive guidance that significantly differs from both informative guidance and no guidance which together form the base case.

\section{Results}

Results obtained show that the regression model was a good fit (R2adj $=.56$ ), and that overall relationships were significant (F3, 18 $=30.73, p<0.001$ ). With other variables held constant, reliance was significantly and positively related to perceived usefulness, perceived task difficulty, and suggestive decisional guidance. The results of the regression analysis are contained in table 6.

Hypothesis 1 is supported. Perceived task difficulty is shown to be a significant and positive predictor of reliance, and therefore the persuasiveness of the decision aid. $(p=.006)$.

Hypothesis 2 is supported. The perceived usefulness of the decision aid was shown to be a significant and positive predictor of reliance, and therefore the persuasiveness of the decision aid $(p<.001)$. 


\begin{tabular}{|c|c|c|c|c|c|}
\hline \multicolumn{6}{|c|}{ Panel A Descriptive Statistics } \\
\hline & \multicolumn{2}{|l|}{ Mean } & Std Deviation & \begin{tabular}{|l} 
Theoretical \\
Range
\end{tabular} & $\begin{array}{l}\text { Observed } \\
\text { range }\end{array}$ \\
\hline Reliance & \multicolumn{2}{|l|}{4.67} & 1.15 & $1-7$ & $1-7$ \\
\hline $\begin{array}{l}\text { Perceived Task Diffi- } \\
\text { culty }\end{array}$ & \multicolumn{2}{|l|}{4.53} & .938 & $1-7$ & $2-6.7$ \\
\hline Perceived usefulness & \multicolumn{2}{|l|}{4.87} & 1.68 & $1-7$ & $1-7$ \\
\hline \multicolumn{6}{|c|}{ Panel B Model Summary } \\
\hline $\mathrm{R}$ & R Square & \multicolumn{2}{|c|}{ Adjusted R Square } & \multicolumn{2}{|c|}{ Std. Error of the Estimate } \\
\hline .763 & .583 & \multicolumn{2}{|l|}{.564} & \multicolumn{2}{|l|}{.761} \\
\hline \multicolumn{6}{|l|}{ Panel C ANOVA } \\
\hline \multicolumn{2}{|l|}{ Sum of Squares } & df & Mean Square & $F$ & Sig. \\
\hline Regression & 53.385 & 3 & 17.795 & 30.725 & $.000^{\mathrm{a}}$ \\
\hline Residual & 38.225 & 66 & .579 & & \\
\hline Total & 91.610 & 69 & & & \\
\hline \multicolumn{6}{|l|}{ Panel D Coefficients } \\
\hline & \multicolumn{2}{|c|}{$\begin{array}{l}\text { Unstandardized Coeffi- } \\
\text { cients }\end{array}$} & \begin{tabular}{|l}
$\begin{array}{l}\text { Standardized } \\
\text { cients }\end{array}$ \\
\end{tabular} & \multirow[t]{2}{*}{$\mathrm{t}$} & \multirow[t]{2}{*}{ Sig. } \\
\hline & $B$ & Std. Error & Beta & & \\
\hline (Constant) & -.039 & .580 & & -.067 & .947 \\
\hline $\begin{array}{l}\text { Perceived task diffi- } \\
\text { culty }\end{array}$ & .280 & .099 & .228 & 2.835 & .006 \\
\hline Perceived usefulness & .659 & .074 & .730 & 8.875 & .000 \\
\hline Suggestive guidance & .697 & .201 & .286 & 3.466 & .001 \\
\hline
\end{tabular}

Hypothesis 3 is supported. Suggestive decisional guidance was shown to be a significant predictor of reliance, and therefore the persuasiveness of the decision aid $(p=.001)$.

Sensitivity testing was conducted to verify the decisional guidance effect; the regression was re-run substituting both informative guidance and no guidance as the predictor variable in lieu of suggestive guidance. The results obtained confirm that both informative guidance $(p=.090)$ or no guidance $(p=.148)$ do not significantly affect reliance, and therefore, in contrast to suggestive guidance, their persuasiveness value is limited.

\section{Discussion}

This study explored factors contributing to the persuasiveness of a decision aid. The empirical results confirm that the characteristics of the target and source of a message, and the design of the message itself, explain a significant proportion of the variation observed. Decision makers were persuaded to reply more on the decision aid when perceived task difficulty and the perceived usefulness of the decision support increased. In line with expectations, the persuasiveness of the decision aid is shown to change when the target and source of the message change. This finding is interesting as it demonstrates the importance of considering these exogenous factors when delivering decision support.

Persuasiveness is enhanced when the message target characteristic in the form the difficulty being experienced by the decision maker increases. This is interrelated also the message source characteristics, where persuasiveness increases in line with how useful the decision maker perceives the system to be for the task at hand. These two character- 
istics would appear to be inextricably linked, with the source and target of the message need to be aligned in order to achieved a persuasive outcomes. Interestingly, additional testing revealed no significant correlation between perceptions of task difficulty and perceptions of usefulness, indicating that the message target and source are in fact independent and separable characteristics.

The characteristics of the message itself resonated well in terms of persuasiveness effects. Providing suggestive guidance increased persuasiveness of the decision aid, by contrast no effect was detected where informative guidance or no guidance was provided. This finding establishes the importance of carefully considering the design of guidance offered by a decision aid. This finding also contributes to the discussion on design of recommender agents, and addresses in part the question posed by Xiao and Benbasat (2007) in relation to the usefulness and impact of explanations in those artifacts.

Persuasiveness was positively enhanced only when suggestive guidance was provided. It may be that the effectiveness of suggestive guidance is related to the novice level participants. Where a suggestion as to how to proceed is offered novices are likely to see this as an acceptable effort accuracy trade-off, and act on the suggestion provided.

By contrast, providing additional information cues for novice users potentially caused an information overload effect that negatively altered their effort accuracy trade-off (by requiring extra effort to process additional cues without increasing accuracy, due to novices limited ability to obtain leverage from additional cues). The case of providing no decisional guidance is interesting also, not overtly considering and incorporating decisional guidance resulted in reduced persuasiveness, illustrating the potential for negative consequences where decisional guidance is absent or inadvertent.

\section{Implications for future research}

The study examined the research question: "What impacts the persuasiveness of deci- sion aids?" with the intention of extending the existing literature relates to persuasiveness in information systems. The study explored the effect of target, source and message characteristics on persuasiveness, the results indicate that the explanatory power of these factors is high, suggesting that further exploration of these constructs will be of value in future research.

Increased understanding of whether and where it is possible to improve the persuasiveness of decision aids will help to extend the existing decision support literature; acting to link more closely the existing behavioral and technical perspectives explored in this literature.

The findings present a starting point for further work to explore more fully the antecedents of persuasiveness. In particular this study establishes direct effects of message characteristics. Future research examining the interrelationships between these message characteristics would more solidly ground current understanding.

\section{Contributions for practice}

The practical implications drawn from this study relate to means of improving the persuasiveness of decision aids. Organizations spend time and money creating these systems with the intention of improving decision outcomes; however no improvement can take place unless decision-makers are persuaded to rely on the decision aid. Improving persuasion levels of decision aids will return additional value to the organization by improving decision quality.

The results show persuasion is a function of the characteristics of the target, source, and message, and these should be taken into account when constructing a decision aid. In particular, practitioners should be mindful of the fact that systems success in decision support extends beyond the design of the tool itself. Successful decision support also requires careful consideration of the characteristics of the decision makers who will be supported. For a difficult task, when the decision support is seen as useful and appropriate 
guidance is provided decision makers will be persuaded to rely on the support offered.

\section{Limitations}

In common with all experimental research this study has several limitations. Small cell sizes may act to limit the explanatory power of the tests undertaken. Data was collected in a laboratory based experimental setting, which maximized the ability to control the environment but introduced some limitations in terms of the richness of the experience for participants. Because of this behavior of participants in a real world setting may differ. The experimental session and data identify only short term effects; a longitudinal study may result in different outcomes. Given the specific problem domain generalizability of the results may be limited, although these results will generalize to any non-normative judgment task which contains similar characteristics to insolvency decision-making. There is also a possibility that the results obtained may relate only to the specific task and software artifact in use.

\section{References}

Arnold, V., et al. "Explanation Provision and Use in an Intelligent Decision Aid," International Journal of Intelligent Systems in Accounting, Finance \& Management (12:1) 2004a, pp 5 - 28.

Arnold, V., et al. "Impact of Intelligent Decision Aids on Experienced and Novice Decision-Makers' Judgments," Accounting \& Finance (44:1) 2004b, pp 1 $-26$.

Arnold, V., and Sutton, S.G. "The Theory of Technology Dominance: Understanding the Impact of Intelligent Decision Aids on Decision Makers' Judgments," Advances in Accounting Behavioral Research (1) 1998, pp 175 - 194.

Collier, P.A., et al. "A validated expert system for decision making in corporate recovery," International Journal of Intelligent Systems in Accounting, Finance \& Management (8) 1999, pp 75 - 88.
Davern, M.J., and Kauffman, R.J. "Discovering potential and realizing value from information technology investments," Journal of Management Information Systems (16:4) 2000, pp 121 - 143.

Davis, F.D. "Perceived usefulness, perceived ease of use, and user acceptance of information technology.," MIS Quarterly (13:3) 1989, pp 319-339.

Davis, F.D., et al. "User acceptance of computer technology: A comparison of two theoretical models," Management Science (35:8) 1989, pp 982 - 1003.

Hampton, C. "Determinants of Reliance: An empirical test of the theory of technology dominance," International Journal of Accounting Information Systems (6) 2005, pp 214 - 240.

Hovland, C.I. Order of presentation in persuasion Yale University Press, New Haven, Conn., 1957.

Hovland, C.I., et al. Communication and persuasion Greenwood Press, Westport, Conn, 1982.

Jiang, J.J., and Klein, G. "Side effects of decision guidance in decision support systems," Interacting with Computers (12) 2000, pp 469 - 481.

Jiang, J.J., et al. "Persuasive expert systems: the influence of confidence and discrepancy," Computers in Human Behavior (16) 2000, pp 99 - 109.

Johnson, E.J., and Payne, J.W. "Effort and Accuracy in Choice," Management Science (31:4) 1985, pp 395 - 414.

Leech, S.A., et al. "A generalized model of decision-making processes for companies in financial distress," Accounting Forum (23:2) 1999, pp 155 - 174.

Leech, S.A., et al. "Impact of intelligent decision aids on human knowledge acquisition," Australian Research Council Discovery Project Grant DP 0344241.

Limayem, M., and DeSanctis, G. "Providing Decisional Guidance for Multicriteria Decision Making in Groups," Infor- 
Persuasive Decision Support: Improving Reliance on Decision Aids / Alison Parkes

mation Systems Research (11:4) 2000, pp 386 - 401.

Mahoney, L.S., et al. "An investigation of the effects of decisional guidance and cognitive ability on decision-making involving uncertainty data," Information \& Organization (13) 2003, pp $85-110$.

Mathieson, K. "Predicting User Intentions: Comparing the Technology Acceptance Model with the Theory of Planned Behavior'," Information Systems Research (2:3) 1991, pp 173 191.

Mazzotta, I., et al. "Portia: a user-adapted persuasion system in the healthy eating domain.," IEEE Intelligent systems (22:6) 2007, pp 42 - 51.

Midden, C., et al. "Using persuasive technology to encourage sustainable behavior," Pervasive Persuasive Technology and Environmental Sustainability Workshop; held at the 6th International Conference on Pervasive Computing, Sydney, Australia, 2008.

Montazemi, A.R., et al. "On the effectiveness of decisional guidance," Decision Support Systems (19) 1996, pp 181 198.

Parikh, M., et al. "The Effectiveness of Decisional Guidance: An Empirical Evaluation," Decision Sciences (32:2) 2001, pp $303-331$.

Payne, J.W., et al. "The Use of Multiple Strategies in Judgment and Choice," in: Individual and Group Decision Making, N.J.J. Castellan (ed.), Lawrence Erblaum Associates, New Jersey, 1993, pp. 19 - 39.

Silver, M.S. "Descriptive Analysis for Computer-Based Decision Support," Operations Research (36:6) 1988, pp 904 916.

Silver, M.S. "Decision Support Systems: Directed and Nondirected Change," In- formation Systems Research (1:1) 1990, pp $47-70$.

Silver, M.S. "Decisional Guidance for Computer-Based Decision Support," MIS Quarterly (15:1) 1991, pp 105 - 122.

Simon, H.A. "A Behavioral Model of Rational Choice," The Quarterly Journal of Economics (69:1) 1955, pp 99 - 118.

Todd, P., and Benbasat, I. "The Use of Information in Decision Making: An Experimental Investigation of the Impact of Computer-Based Decision Aids," MIS Quarterly (16:3) 1992, pp 373 - 393.

Todd, P., and Benbasat, I. "The Influence of Decision Aids on Choice Strategies: An Experimental Analysis of the Role of Cognitive Effort," Organizational Behavior and Human Decision Processes (60) 1994, pp 36 - 74.

Todd, P., and Benbasat, I. "Evaluating the Impact of DSS, Cognitive Effort, and Incentives on Strategy Selection," Information Systems Research (10:4) 1999, pp 356 - 374.

Van de Ven, A.H., and Delbecq, A.L. "A Task Contingent Model of Work-Unit Structure," Administrative Sciences Quarterly (19:2) 1974, pp 183-197.

Van de Ven, A.H., and Ferry, D.L. "Measuring and assessing organizations," John Wiley \& Sons, New York, 1980.

Wilson, E.V., and Zigurs, I. "Decisional Guidance and end-user display choices," Accounting, Management \& Information Technology (9) 1999, pp 49 75.

Wood, R.E. "Task Complexity: Definition of the Construct," Organizational Behavior and Human Decision Processes (37) 1986, pp 60 - 82.

Xiao, B., and Benbasat, I. "E-Commerce Product Recommendation Agents: Use, Characteristics and Impact," MIS Quarterly (31:1) 2007, pp 137-209. 


\section{About the Authors}

Dr Alison Parkes is a Senior Lecturer in the Faculty of Business and Economics at the University of Melbourne. She holds a Bachelor degree in Commerce (Accounting), Honors and Masters degrees in Information Systems, and a PhD from the University of Melbourne in Business Information Systems. Prior to entering academia she gained considerable practical experience in both the public and private sectors, having held senior level appointments in a range of accounting and information technology roles. She has considerable expertise in the contextual design and implementation of financial systems and currently consults in this area. Her research explores various forms of human computer interactions, with a particular interest in the performance implications and behavioral consequences of technology design choices. Alison Parkes is the corresponding author and can be contacted at: aparkes@unimelb.edu.au 